\title{
Delivery latency and placental cord insertion in preterm labor with singleton pregnancy
}

Soo Ran Choi, Hyung Eun Choi, Eunae Jo, Hwayeon Choi, Haesun Joo, Su Kyung Jung, Shina Jang, Jung Ok Kim, Sung Ook Hwang Department of Obstetrics and Gynecology, Inha University College of Medicine, Inha University Hospital, Incheon, Korea

\section{Problem statement}

Marginal and velamentous cord insertion are associated with increased risk of spontaneous preterm birth (PTB) that includes preterm labor (PTL) and preterm premature rupture of membranes (PPROM). A shorter transvaginal cervical length $(\leq 2 \mathrm{~cm})$ and amniotic fluid index $(\leq 5 \mathrm{~cm})$ independently predict delivery within 7 days in PPROM. The aim of this study was to determine whether peripheral insertion of umbilical cord (PIUC) is associated with short delivery latency in PTB with PTL and PPROM.

\section{Methods}

This study was conducted retrospectively in delivered women with singleton pregnancy between 24-42 weeks of gestation. 110 women were delivered $<37$ weeks and 112 women were delivered at term ( $\geq 37$ weeks). Velamentous cord insertion, twin, placenta previa, abruptio placenta, fetal anomaly, uterine anmaly, and PTB with medical and surgical indications were exlcuded. PIUC was definded $\leq 3 \mathrm{~cm}$ from placental edge. Multiple regression analysis was used for statistical analysis.

\section{Results}

\section{Comparison of placental characteristics}

\begin{tabular}{lccc} 
& $\begin{array}{c}\text { PTB } \\
(\mathbf{n}=110)\end{array}$ & $\begin{array}{c}\text { Term } \\
(\mathrm{n}=112)\end{array}$ & $P$-value \\
\hline PI. to birth weight ratio & $0.26 \pm 0.07$ & $0.19 \pm 0.03$ & $<0.001$ \\
PI. the longest length $(\mathrm{cm})$ & $17.87 \pm 2.79$ & $19.86 \pm 2.19$ & 0.691 \\
& & & \\
PI. the shortest length $(\mathrm{cm})$ & $15.54 \pm 2.68$ & $17.13 \pm 2.01$ & 0.147 \\
PI. thickness (cm) & $2.06 \pm 0.57$ & $2.25 \pm 0.59$ & 0.119 \\
Cord insertion $(\mathrm{cm})$ & $4.02 \pm 2.28$ & $4.96 \pm 2.57$ & 0.207 \\
PIUC $(\%)^{*}$ & $26(40.6 \%)$ & $24(36.4 \%)$ & 0.940 \\
Cord length $(\mathrm{cm})$ & $38.70 \pm 12.70$ & $43.49 \pm 12.08$ & 0.354 \\
Cord thickness $(\mathrm{cm})$ & $1.27 \pm 0.45$ & $1.10 \pm 0.27$ & 0.069 \\
\hline
\end{tabular}

Multiple regression analysis between factors and delivery latency

\begin{tabular}{lcc}
\hline \multicolumn{1}{c}{ Factors } & $\boldsymbol{\beta} \pm \mathrm{SE}$ & $\boldsymbol{P}$-value \\
\hline Cord insertion & $3.551_{ \pm} 1.439$ & 0.017 \\
Age & $-0.183_{ \pm} 0.571$ & 0.750 \\
Parity & $-5.455_{ \pm} 4.009$ & 0.180 \\
Cx length & $1.355_{ \pm} 3.270$ & 0.680 \\
Cx dilatation & $-1.628_{ \pm} 1.732$ & 0.349 \\
AFI & $0.435_{ \pm} 0.571$ & 0.449 \\
CRP & $-0.319_{ \pm} 2.145$ & 0.882 \\
Placenta to birth weight ratio & $-0.050_{ \pm} 37.162$ & 0.999 \\
PI. the longest length & $1.447_{ \pm} 1.158$ & 0.217 \\
PI. thickness & $11.000_{ \pm} 5.319$ & 0.044 \\
Cord length & $-0.114_{ \pm} 0.250$ & 0.649 \\
Cord thickness & $6.734_{ \pm} 6.664$ & 0.317 \\
\hline
\end{tabular}

\section{Clinical characteristics in PTB}

\begin{tabular}{lcccc}
\hline & PTB $(\mathbf{n}=110)$ & PTL $(\mathbf{n}=54)$ & $\begin{array}{c}\text { PPROM } \\
(\mathbf{n}=56)\end{array}$ & P-value \\
\hline Age (yrs) & $31.67 \pm 5.16$ & $30.47 \pm 5.51$ & $32.64 \pm 4.88$ & 0.099 \\
Parity & $0.61 \pm 0.70$ & $0.44 \pm 0.62$ & $0.73 \pm 0.76$ & 0.068 \\
Adm GA (weeks) & $30.86 \pm 3.89$ & $29.72 \pm 4.12$ & $31.90 \pm 3.43$ & 0.026 \\
Del GA (weeks) & $32.06 \pm 3.35$ & $31.87 \pm 3.52$ & $32.88 \pm 2.80$ & 0.210 \\
Delivery latency (day) & $11.41 \pm 24.63$ & $16.63 \pm 31.13$ & $6.67 \pm 15.77$ & 0.122 \\
Adm Cx length (cm) & $0.83 \pm 1.06$ & $0.52 \pm 0.83$ & $1.11 \pm 1.18$ & 0.026 \\
Cx dilatation (cm) & $1.78 \pm 2.11$ & $2.17 \pm 2.39$ & $1.42 \pm 1.78$ & 0.164 \\
AFI (cm) & $9.87 \pm 5.14$ & $11.78 \pm 5.26$ & $8.13 \pm 4.44$ & 0.004 \\
CRP (mg/dl) & $0.77 \pm 1.33$ & $0.76 \pm 1.44$ & $0.77 \pm 1.25$ & 0.988 \\
Infant weight(g) & $2006.4 \pm 684.0$ & $1996.7 \pm 723.9$ & $2098.2 \pm 594.9$ & 0.544 \\
pl. weight (g) & $482.3 \pm 151.8$ & $490.0 \pm 156.8$ & $485.4 \pm 141.4$ & 0.902 \\
pl. to birth weight ratio & $0.26 \pm 0.07$ & $0.26 \pm 0.08$ & $0.24 \pm 0.06$ & 0.269 \\
pl. the longest length (cm & $17.87 \pm 2.79$ & $18.23 \pm 2.83$ & $17.62 \pm 2.74$ & 0.387 \\
pl. thickness (cm) & $2.06 \pm 0.57$ & $2.02 \pm 0.48$ & $2.07 \pm 0.66$ & 0.718 \\
Cord insertion (cm) & $4.02 \pm 2.28$ & $4.35 \pm 2.33$ & $3.63 \pm 2.17$ & 0.209 \\
PIUC (\%) & $40.60 \%$ & $30.00 \%$ & $51.50 \%$ & 0.083 \\
Cord length (cm) & $38.70 \pm 12.70$ & $37.43 \pm 11.82$ & $40.15 \pm 13.37$ & 0.398 \\
Cord thickness (cm) & $1.27 \pm 0.45$ & $1.33 \pm 0.46$ & $1.22 \pm 0.42$ & 0.337 \\
\hline
\end{tabular}

\section{Comparison between PIUC and non PIUC in PTB}

\begin{tabular}{lccc}
\hline & $\begin{array}{c}\text { PIUC } \\
(\leq 3 \mathrm{~cm}, \mathrm{n}=47)\end{array}$ & $\begin{array}{c}\text { Non PIUC } \\
(>3 \mathrm{~cm}, \mathrm{n}=63)\end{array}$ & P-value \\
\hline Age (yrs) & $31.62 \pm 6.03$ & $31.59 \pm 4.63$ & .988 \\
Parity & $0.46 \pm 0.65$ & $0.65 \pm 0.75$ & .308 \\
Delivery latency (day) & $3.00 \pm 3.68$ & $9.82 \pm 17.2$ & .031 \\
Cx length (cm) & $1.03 \pm 1.21$ & $0.68 \pm 0.93$ & .198 \\
Cx_dilatation (cm) & $1.73 \pm 2.4$ & $1.81 \pm 1.91$ & .881 \\
AFI (cm) & $8.66 \pm 5.1$ & $10.72 \pm 5.07$ & .119 \\
CRP (mg/dl) & $0.88 \pm 1.89$ & $0.69 \pm 0.75$ & .588 \\
Infant weight (g) & $1937.69 \pm 547.25$ & $2128.65 \pm 719.55$ & .259 \\
PI. weight (g) & $474.04 \pm 121.89$ & $497.08 \pm 164.45$ & .547 \\
Pl. to birth weight ratio & $0.26 \pm 0.07$ & $0.25 \pm 0.08$ & .692 \\
Pl. the longest length (cm) & $17.25 \pm 2.35$ & $18.38 \pm 2.99$ & .113 \\
PI. the shortest length (cm) & $2.18 \pm 0.49$ & $1.95 \pm 0.62$ & .126 \\
Cord length (cm) & $37.21 \pm 12.02$ & $40.01 \pm 13.08$ & .390 \\
Cord thickness (cm) & $1.19 \pm 0.39$ & $1.33 \pm 0.47$ & .219 \\
\hline
\end{tabular}

\section{Conclusion}

PIUC was associated shorter delivery latency in preterm singleton pregnancy. Placental cord insertion site should be evaluated at ultrasonographic examination of women with preterm labor. 